\title{
Algorithm construction methodology for diagnostic classification of near-infrared spectroscopy data
}

\author{
Ramón Guevara ${ }^{\mathrm{a}, \mathrm{b}, \mathrm{c}}$, Lynn Stothers ${ }^{\mathrm{a}, \mathrm{b}}$ and Andrew Macnab ${ }^{\mathrm{a}, \mathrm{d}, *}$ \\ ${ }^{a}$ Department of Urology, Faculty of Medicine, University of British Columbia, Canada \\ ${ }^{\mathrm{b}}$ UBC Hospital Bladder Care Centre, Vancouver, BC, Canada \\ ${ }^{\mathrm{c}}$ Basque Center on Cognition, Brain and Language, Donostia, Spain \\ ${ }^{\mathrm{d}}$ Stellenbosch Institute for Advanced Study, Wallenberg Research Centre, Stellenbosch University, \\ Stellenbosch, South Africa
}

\begin{abstract}
Background: Near-infrared spectroscopy (NIRS) has recognized potential but limited application for non-invasive diagnostic evaluation. Data analysis methodology that reproducibly distinguishes between the presence or absence of physiologic abnormality could broaden clinical application of this optical technique.

Methods: Sample data sets from simultaneous NIRS bladder monitoring and invasive urodynamic pressure-flow studies (UDS) are used to illustrate how a diagnostic algorithm is constructed using classification and regression tree (CART) analysis. Misclassification errors of CART and linear discriminant analysis (LDA) are computed and examples of other urological NIRS data likely amenable to CART analysis presented.

Results: CART generated a clinically relevant classification algorithm (error 4\%) using 46 data sets of changes in chromophore concentration composed of the whole time series without specifying features. LDA did not (error 16\%). Using CART NIRS data provided comparable discriminant ability to the UDS diagnostic nomogram for the presence or absence of obstructive pathology ( $88 \%$ specificity, $84 \%$ precision). Pilot data examples from children with and without voiding dysfunction and women with mild or severe pelvic floor muscle dysfunction also show potentially diagnostic differences in chromophore concentration.

Conclusions: CART analysis can likely be applied in other NIRS monitoring applications intended to classify patients into those with and without pathology.

Keywords: Classification and regression tree (CART), diagnostic algorithm, linear discriminant analysis, near-infrared spectroscopy, urodynamics
\end{abstract}
Abbreviations
BOO Bladder outlet obstruction;
CART Classification and regression tree;
ICA Independent component analysis;
$\mathrm{HHb}$ Deoxy-hemoglobin;
$\mathrm{O}_{2} \mathrm{Hb}$ Oxy-hemoglobin;
LDA Linear discriminant analysis;

\footnotetext{
${ }^{*}$ Corresponding author: Andrew Macnab, MD, FRCPC, FRCPCH, FCAHS, UBC Hospital Bladder Care Centre, Unit 1B, Room F329, 2211 Wesbrook Mall, Vancouver, BC, V6T 2B5 Canada. Tel.: +1 604 822 7616; Fax: +1 604822 7591; E-mail: amacnab@cw.bc.ca.
} 
LUTS Lower urinary tract symptoms;

NIRS Near-infrared spectroscopy;

NLUTD Non-neurogenic lower urinary tract dysfunction;

PCA Principal component analysis;

$\mathrm{tHb}$ Total hemoglobin;

UDS Urodynamic pressure-flow studies.

\section{Introduction}

Recent detailed reviews summarize the evolution of near-infrared spectroscopy (NIRS) and describe the basic principles, instrumentation, advantages and limitations of this optical technique in the context of multiple research and clinical applications [1,2,7,11,25,34,35]. Methods have been developed to quantify a significant number of oxygenation and hemodynamic parameters [34] and successfully monitor a variety of tissues and organs $[1,2,7,15,25,34,35]$. Initial research using NIRS predominantly investigated cerebral oxygenation $[7,15,25,34,35]$ and a major current application is the study of skeletal muscle metabolism [1,2,7,11,34]. Research applications predominate [7,15,25,34], but examples of methodologies with clinical relevance are reported [3,9,15,21,24,30,35] including non-invasive investigation of voiding dysfunction in adults [18,31,32] and children [27]. However, while the ability to employ NIRS clinically is recognized to be attractive for many reasons $[9,11,15,32,34,35]$ to become clinically relevant further studies are required to define NIRS parameters associated with normal and pathological function, confirm the reproducibility of NIRS data and diagnostic criteria, and define analysis methodology that enables NIRS monitoring to be used to answer clinical questions [9,23,34].

The principle chromophores of interest in NIRS monitoring of human tissue are the oxygenated and reduced forms of hemoglobin, $\mathrm{O}_{2} \mathrm{Hb}$ and $\mathrm{HHb}$, respectively [7,34]. In many monitoring applications it is the pattern and magnitude of change in $\mathrm{O}_{2} \mathrm{Hb}$ and $\mathrm{HHb}$ that are of principal interest $[7,23,25,34]$. Even though this data provides changes in chromophore concentration from baseline, rather than quantified values $[7,15,34]$ the well-recognized patterns of change occurring in response to known physiologic events such as hypoxia, ischemia and changes in blood volume can be of considerable clinical value $[3,7,11,15,25]$. Hence, NIRS monitoring allows novel insights into changes in tissue oxygenation and hemodynamics $[2,34,35]$, and in many clinical situations such data is unique as it cannot readily be obtained by other means.

A recent example is the data obtained using NIRS to monitor the changes in $\left[\Delta \mathrm{O}_{2} \mathrm{Hb}\right],[\Delta \mathrm{HHb}]$, and total hemoglobin $(\mathrm{tHb})$ that occur in the bladder detrusor muscle as the organ empties during the voiding cycle $[18,32]$. Such changes in chromophore concentration monitored transcutaneously during simultaneous NIRS and invasive urodynamic pressure-flow studies (UDS) are recognized to provide physiologic information related to bladder oxygenation and hemodynamics $[18,23]$. NIRS has relevance as a method for investigating bladder dysfunction because chronic pressure elevation within the urinary tract is recognized to cause pathological changes which alter the dynamics of bladder blood flow and oxygen supply and demand [10]. Also, because the non-invasive nature of the technique is attractive to patients who have low tolerance for the catheterization UDS involves. However, in spite of the desirability of NIRS evaluation of bladder dysfunction, as with other applications using NIRS, such monitoring requires data analysis methodology that enables clinicians to reliably distinguish between normal and pathological states in order to be relevant clinically.

In this context an algorithm was initially developed that incorporated NIRS-derived changes in chromophore concentration to classify patients for the presence or absence of bladder outlet obstruction 
(BOO) and the ability of this algorithm compared to the 'gold standard' of UDS classification using pressure-flow data applied to the Abrams-Griffiths nomogram [14]. The algorithm combined three noninvasive parameters: (1) the positive or negative trend in $\mathrm{O}_{2} \mathrm{Hb}$ during voiding, (2) maximum urine flow rate $\left(Q_{\max }\right)$ and (3) residual urine volume (PVR). This algorithm had comparable discriminant ability to UDS classification for the presence or absence of BOO in symptomatic men [18]. Recently similar diagnostic accuracy was obtained just using changes in $\left[\Delta \mathrm{O}_{2} \mathrm{Hb}\right]$ and $[\Delta \mathrm{HHb}]$ and an algorithm generated by mathematical modeling and Classification and Regression Tree (CART) analysis [31].

As we believe this method of analysis could be applicable in other research and clinical applications using NIRS monitoring, this paper describes the principles and process required to apply CART analysis to NIRS data and develop a diagnostic algorithm. Data sets from the NIRS diagnostic method for BOO are used as examples, and additional data presented that also appears ideally suited to CART analysis and construction of diagnostic algorithms intended to classify patients into those with and without pathology.

\section{Materials and methods}

\subsection{Participants and instruments}

The NIRS data sets used as examples were obtained from a cohort of male subjects presenting with lower urinary tract symptoms (LUTS) evaluated using simultaneous UDS and NIRS monitoring, as described previously [31]. The sensor patch of a laser-powered NIRS instrument with 3 wavelengths (785, 808 and $830 \mathrm{~nm}$ ) was applied to the skin to locate the optodes $2 \mathrm{~cm}$ above the pubic bone across the midline over the bladder [17]. The source-detector separation was $4 \mathrm{~cm}$ to optimize penetration of photons to the anterior wall of the bladder and the detrusor muscle. Data was collected at $6 \mathrm{~Hz}$ and a conventional algorithm used to generate chromophore concentrations from raw optical density changes, and real-time graphic display of $\mathrm{O}_{2} \mathrm{Hb}, \mathrm{HHb}$ and tHb [17].

Additional data to further illustrate the potential relevance of CART-derived diagnostic algorithms comes from two pilot studies where the number of participants is insufficient as yet for CART analysis. In pilot 1 asymptomatic children and a cohort requiring investigation for a common form of voiding dysfunction, non-neurogenic lower urinary tract dysfunction (NLUTD), were monitored using NIRS [16] during voluntary bladder emptying (natural voiding) [27]. NLUTD is currently poorly understood in terms of the physiology underlying these children's symptoms and difficult to diagnose. The NIRS instrument was a miniature wireless device [28] positioned on the abdominal skin in the same location as the optode patch of the laser-powered device [17]. The light source has 3 paired light emitting diodes with wavelengths of 760 and $850 \mathrm{~nm}$ separated from a silicon photodiode to provide source-detector separation distances of 3, 3.5 and $4 \mathrm{~cm}$. Data was collected at $10 \mathrm{~Hz}$. In pilot 2 muscle function in women with varying degrees of pelvic floor dysfunction was evaluated during voluntary contractions, using a laser-powered NIRS instrument (Oxymon, Artinis BV, The Netherlands), [33] with a vaginal probe that incorporates a NIRS emitter and 2 sensors [29]. Monitoring data was converted to graphs of change in chromophore concentration by proprietary software; the same algorithm was used in both NIRS instruments [33]. The patterns of change and trends in $\mathrm{O}_{2} \mathrm{Hb}, \mathrm{HHb}$ and tHb during voiding (pilot 1) and pelvic floor contraction (pilot 2) were compared between normal and symptomatic subjects.

\subsection{Data analysis}

Urodynamic data was first analyzed using standard UDS methodology [22]; patients were classified for the presence or absence of bladder outlet obstruction by plotting the values of maximum urine 
flow rate $Q_{\max }$ and detrusor pressure at maximum flow rate $P_{\text {det }}\left(Q_{\max }\right)$ on the Abrams-Griffiths nomogram [14].

NIRS data from these patients was then analyzed using two different classification techniques: linear discriminant analysis (LDA) [6] and classification and regression trees (CART) $[4,8,20]$ and the methods compared. Such classification is used to predict values of a categorical outcome (dependent variable) from one or more predictor (independent) variables.

LDA is a classic method of classification that projects high-dimensional data onto a low-dimensional sub-space where the data achieves maximum class separability [6]. LDA performs classification by generating linear combinations of the predictor variables onto which the data set can be projected and visualized as axes transformations. The optimal projection is obtained by minimizing the within-class distance, while maximizing the between-class distance. Maximum separability is achieved by linear combinations that rotate the axes in such a way that the projection of the data on the new axes is minimally spatially mixed.

CART is a tree-building classification methodology that considers all possible splits of all variables, and is ideally suited for clinical applications where variables are often not normally distributed and large variations may occur between different patients [4]. CART analysis requires a learning set of data that contains the values of the predictor variables for which the categorical outcome is known. The CART algorithm generates a recursive tree which best fits the data of the learning set that can then be used for the classification of unknown data. CART is well-established and widely applied mathematical modeling and analysis method, and the steps involved in the process are comprehensively described $[4,8,20]$.

When applying CART to the bladder monitoring data the categorical outcomes are the presence (obstructed) and absence (unobstructed) of $\mathrm{BOO}$, and the predictor variables $\left[\Delta \mathrm{O}_{2} \mathrm{Hb}\right],[\Delta \mathrm{HHb}]$ for NIRS and $P_{\mathrm{det}}, Q$ for UDS. Prior to analysis segments of the NIRS data stream with artifact generated by spontaneous movement is excluded. The tree is then generated via a branching process involving recursive partitioning of the learning set, which is first divided into two parts using one of the predictor variables. Values of $[\Delta \mathrm{HHb}]$ versus $\left[\Delta \mathrm{O}_{2} \mathrm{Hb}\right]$ are plotted for every element in the learning set. A division can be made using a particular value of $[\Delta \mathrm{HHb}]$ or of $\left[\Delta \mathrm{O}_{2} \mathrm{Hb}\right]$ by splitting the region with a line that is parallel to one of the two coordinate axes. The tree is completed when every data point constitutes a one-member category in itself. To provide effective classification of new data this tree is then pruned as it otherwise follows the idiosyncrasies of the original learning set too closely. The optimal tree is a compromise: neither too complex so that it over fits the data, nor excessively pruned and so simple that it under fits the data. The version of the algorithm with the smallest misclassification rate is identified via a test sample or cross-validation estimate. The test sample method uses an independent data set not included in the construct of the initial tree. If such data is not available a cross-validation estimate uses a data set derived by dividing the learning sample into equal subsets. Each subset is then used in turn as a test sample with the remainder of the data used as the learning set.

The LDA and CART algorithms used are included in the MATLAB Statistics Toolbox. Misclassification error is estimated by randomly splitting the dataset (10 splits) into training and validation data. Each validation split is then used to assess the predictive accuracy of the classification model, with the remaining part of the data used for training. The results are then averaged over the splits (10-fold cross-validation).

Using LDA we first classified sets of data points containing the whole time course of voiding (uroflow start to uroflow end). These sets differed on the selected predictor variables (two variables each time). Subsequently, we tried to identify useful features to improve classification. Several features, including 
latencies, variances, mean slopes and several combinations of the predictor variables or important landmarks in the data stream, were rejected because they gave very low predictive accuracy. Ultimately we used one point per patient instead of using the whole time course. The principal feature chosen for classification was $Q_{\max }$ because this is the feature used in the Abrams-Griffiths nomogram, where it gives good results when using urodynamic variables alone. CART analysis was then performed using exactly the same procedure (same sets of data points, with and without features). The objective was to identify if the CART methodology resulted in a lower misclassification error than LDA. Principal component analysis (PCA) [20] and independent component analysis (ICA) [5,13] were also used prior to classification, in an attempt to find possible linear combinations of the variables. PCA transforms a number of correlated variables into a smaller number of uncorrelated variables (principal components). The first principal components account for most of the variability of the data. In a similar fashion, ICA looks for a linear transformation of the data that produce statistically independent components.

\section{Results}

Forty six data sets (30 obstructed and 16 unobstructed) [31] were used to correlate the diagnostic classifications derived from UDS data with NIRS parameters.

Figure 1 shows a sample graph illustrating the characteristic differences between unobstructed patients (normal pathology) and those who have bladder outlet obstruction (abnormal pathology) for uroflow data (voiding), bladder pressure $\left(P_{\mathrm{det}}\right)$, and changes in NIRS-derived chromophore concentrations and tHb plotted against time. In unobstructed subjects a positive trend in $\mathrm{O}_{2} \mathrm{Hb}$ and $\mathrm{tHb}$ gradually begins following permission to void; this increases following the start of uroflow and an overall increase occurs over the course of voiding. In contrast a negative trend is seen in obstructed subjects from permission to void to uroflow end. In obstructed subjects $P_{\text {det }}$ also rises higher and the period required for voiding (uroflow) is greater than in unobstructed subjects.

Tables 1 and 2 summarize the classification. Table 1 shows the results using LDA with $Q_{\max }$ selected as a feature, and Table 2 the performance of the CART algorithm with no feature selected (i.e., data from the whole time course of voiding). The performance of LDA using the same time course is not included because it is not clinically relevant as misclassification errors higher than $70 \%$ resulted for all pairs of NIRS related variables. Similarly the performance of CART using $Q_{\max }$ as a feature is not included as this is indistinguishable from the values reported for LDA at $Q_{\max }$. The error reported in both tables is the 10-fold cross-validation estimate of the misclassification rate of the NIRS-derived diagnosis with respect to urodynamic classification using pressure-flow measurements applied to the Abrams-Griffiths nomogram [14]. Therefore errors of $0 \%$ (Table 2) indicate that the CART algorithm gave the same result as the standard UDS nomogram (not an "absolute" error of $0 \%$ ).

Table 1 shows that using LDA with $[\Delta \mathrm{HHb}],\left[\Delta \mathrm{O}_{2} \mathrm{Hb}\right]$ the miscalculation error was $40.8 \%$. The error was lowest $(15 \%)$ when using $[\Delta \mathrm{HHb}], P_{\text {det }}$ - very close to the $18.3 \%$ error obtained using urodynamic pressure-flow measurements $\left(P_{\text {det }}, Q\right)$ alone. The highest error was $50.7 \%$ when using $\left[\Delta \mathrm{O}_{2} \mathrm{Hb}\right], Q$.

Table 2 shows that using NIRS data alone, $[\Delta \mathrm{HHb}],\left[\Delta \mathrm{O}_{2} \mathrm{Hb}\right]$, CART classification had $88 \%$ specificity, $84 \%$ precision and a miscalculation error of $4.3 \%$; and that the algorithm had the same discriminant ability as the standard UDS nomogram with an error of $0 \%$ whenever $P_{\text {det }}$ was used with any other variable $\left(P_{\mathrm{det}}, Q ;[\Delta \mathrm{HHb}], P_{\mathrm{det}} ;\right.$ and $\left.\left[\Delta \mathrm{O}_{2} \mathrm{Hb}\right], P_{\mathrm{det}}\right)$.

Further examples of changes in chromophore concentration likely amenable to classification using CART analysis are provided by data from two recent pilot studies. When voiding chromophore data 


\section{A- Unobstructed bladder outlet}

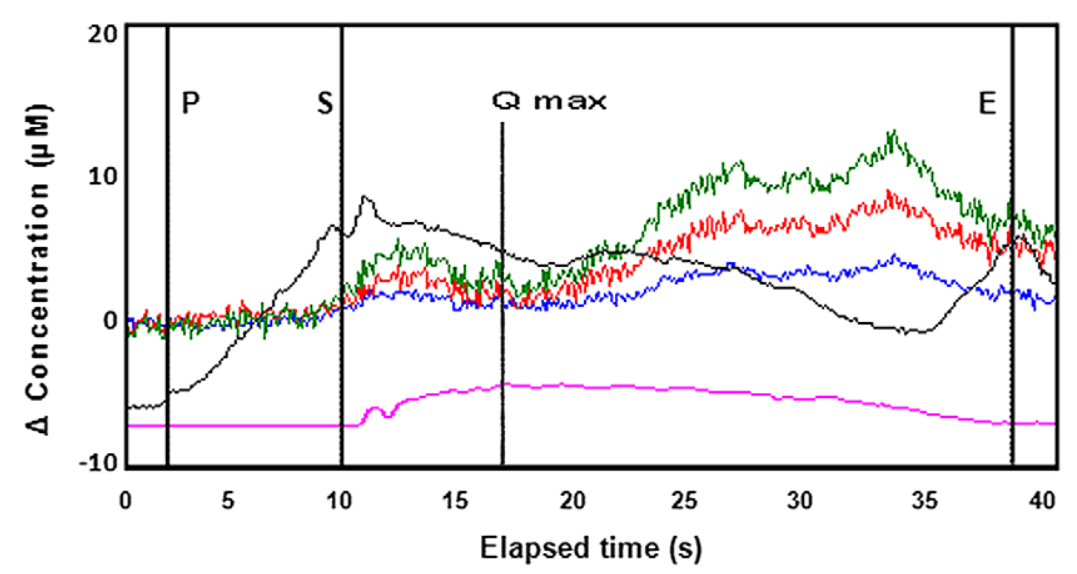

B- Obstructed bladder outlet
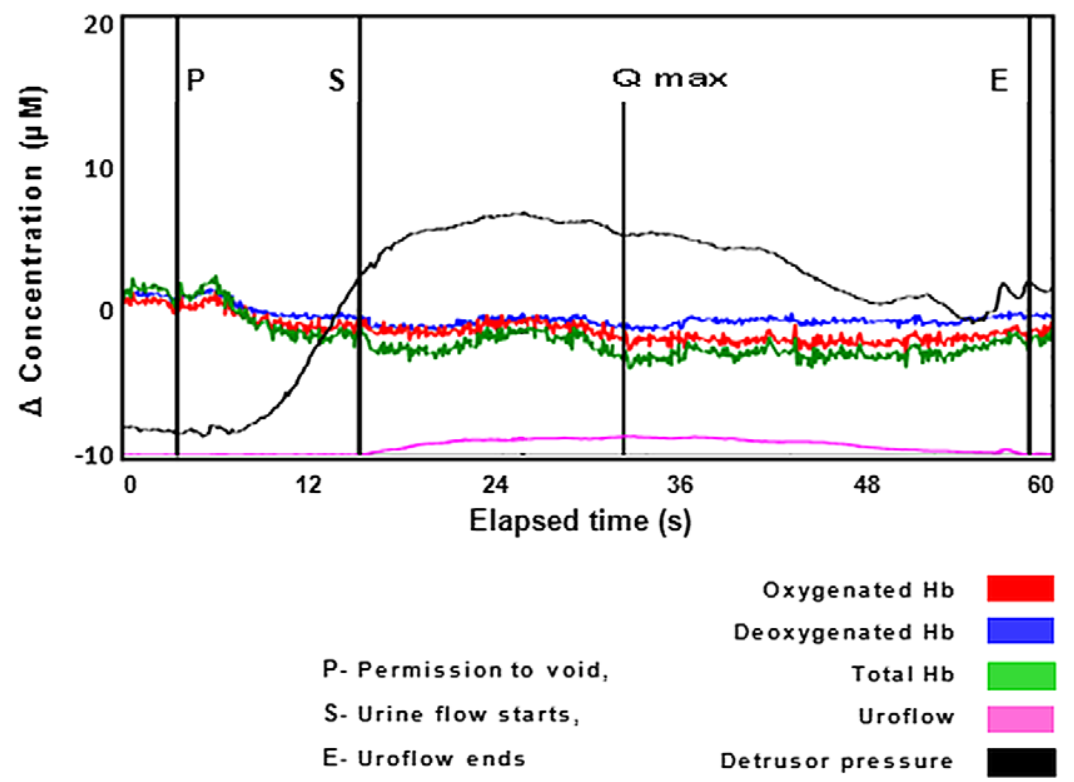

Fig. 1. An example of NIRS data sets from an unobstructed (normal) and obstructed (pathologic) patient with uroflow and detrusor pressure against time. The trend of chromophore concentration from zero for $\mathrm{O}_{2} \mathrm{Hb}, \mathrm{HHb}$ and tHb differs (positive trend when unobstructed, negative trend when obstructed). Five point averaging was used to smooth the NIRS data in such graphs prior to analysis. The start and end of uroflow (voiding) defines the period from which all NIRS data is used in CART analysis. Peak uroflow $\left(Q_{\max }\right)$ is the point providing $P_{\text {det }}$ measurement for UDS classification and the NIRS data set used in LDA classification. (Colors are visible in the online version of the article; http://dx.doi.org/10.3233/SPE-2010-0486.)

from asymptomatic children and those with non-neurogenic lower urinary tract dysfunction (NLUTD) are compared, the positive trend in detrusor $\left[\Delta \mathrm{O}_{2} \mathrm{Hb}\right]$ and $[\mathrm{tHb}]$ seen in asymptomatic children differs to the negative trend evident in children with NLUTD [16,27]. An additional discriminant feature is that $[\Delta \mathrm{HHb}]$ is also greater than $\left[\Delta \mathrm{O}_{2} \mathrm{Hb}\right]$ when symptomatic children void. Comparing data from subjects with pelvic floor muscle dysfunction different magnitudes of changes in chromophore concentration are observed during repeated maximal pelvic floor muscle contractions between those with mild and severe symptoms (Fig. 2). In addition a difference is evident in the slope of recovery of $\mathrm{O}_{2} \mathrm{Hb}$ at the end 
Table 1

Results for the classification using LDA

\begin{tabular}{lccrrrrrr}
\hline Predictor variables $\left(\right.$ at $\left.Q_{\max }\right)$ & Number of patients & Error $(\%)$ & TP & \multicolumn{1}{c}{ TN } & FP & FN & Sensitivity (\%) & Specificity (\%) \\
\hline$[\Delta \mathrm{HHb}],\left[\Delta \mathrm{O}_{2} \mathrm{Hb}\right]$ & 46 & 40.8 & 17 & 10 & 6 & 13 & 56.7 & 62.5 \\
{$[\Delta \mathrm{HHb}], Q$} & 46 & 49.5 & 26 & 2 & 14 & 4 & 86.7 & 12.5 \\
{$\left[\Delta \mathrm{O}_{2} \mathrm{Hb}\right], Q$} & 46 & 50.7 & 26 & 2 & 14 & 4 & 86.7 & 12.5 \\
$P_{\mathrm{det}}, Q$ & 46 & 18.3 & 21 & 15 & 1 & 9 & 70.0 & 93.8 \\
{$[\Delta \mathrm{HHb}], P_{\text {det }}$} & 46 & 15.0 & 23 & 15 & 1 & 7 & 76.7 & 93.8 \\
{$\left[\Delta \mathrm{O}_{2} \mathrm{Hb}\right], P_{\text {det }}$} & 46 & 16.0 & 22 & 15 & 1 & 8 & 73.3 & 93.8 \\
\hline
\end{tabular}

Notes: Pairs of predictor variables are used that are evaluated at the point of maximum flow rate $\left(Q_{\max }\right)$; the ability of the method to correctly classify is given by the "Error", which is the estimate for the misclassification rate of future data, using 10-fold cross-validation. True positive (TP) - the fraction of patients correctly classified as obstructed by the algorithm; true negative $(\mathrm{TN})$ - the fraction of patients correctly classified as unobstructed by the algorithm; false positive (FP) - the fraction of patients classified as obstructed by the algorithm and unobstructed by the Abrams-Griffiths nomogram; [26] false negative (FN) - the fraction of patients classified as unobstructed by the algorithm and obstructed by nomogram. Sensitivity $=\mathrm{TP} /(\mathrm{TP}+\mathrm{FN})$ and Specificity $=\mathrm{TN} /(\mathrm{TN}+\mathrm{FP})$

Table 2

Results for the classification using the CART algorithm

\begin{tabular}{lcccccccc}
\hline Predictor variables & Number of patients & Error $(\%)$ & TP & TN & FP & FN & Sensitivity (\%) & Specificity (\%) \\
\hline$[\Delta \mathrm{HHb}],\left[\Delta \mathrm{O}_{2} \mathrm{Hb}\right]$ & 46 & 4.3 & 30 & 14 & 0 & 2 & 1 & 87.5 \\
{$[\Delta \mathrm{HHb}], Q$} & 46 & 10.9 & 30 & 11 & 5 & 0 & 1 & 68.8 \\
{$\left[\Delta \mathrm{O}_{2} \mathrm{Hb}\right], Q$} & 46 & 17.4 & 30 & 8 & 8 & 0 & 1 & 50.0 \\
$P_{\text {det }}, Q$ & 46 & 0 & 30 & 16 & 0 & 0 & 1 & 1 \\
{$[\Delta \mathrm{HHb}], P_{\text {det }}$} & 46 & 0 & 30 & 16 & 0 & 0 & 1 & 1 \\
{$\left[\Delta \mathrm{O}_{2} \mathrm{Hb}\right], P_{\text {det }}$} & 46 & 0 & 30 & 16 & 0 & 0 & 1 & 1 \\
\hline
\end{tabular}

Notes: Nomenclature is the same as in Table 1. However, unlike in LDA, NIRS data predictor variables are used from the entire voiding interval (uroflow start to uroflow end), not just from the point of maximal flow rate $Q_{\max }$.

of contractions (a measure of reoxygenation), and this and the impression of a negative trend in [tHb] (predominantly due to reduced $\mathrm{O}_{2} \mathrm{Hb}$ ) when muscle dysfunction is severe, suggest the suitability of this data for classification using CART analysis.

\section{Discussion}

CART analysis using changes in NIRS chromophore concentration in the bladder detrusor muscle during voiding has comparable discriminant ability to the current invasive urodynamic pressure-flow methodology when classifying patients for the presence or absence of bladder outlet obstruction [23,31]. The differences evident between pilot data from children with and without NLUTD [16], and women with mild and severe pelvic floor dysfunction, are other examples of NIRS data where CART could be likely be applied to classify patients based on trends or magnitude of chromophore change that differ in health and disease. CART algorithms that incorporate parameters other than NIRS chromophore data can already discriminate between complex data sets in clinical situations involving patients with primary carcinoma [12] and myocardial infarction [19]. Consequently the use of CART analysis could well benefit other investigators using NIRS monitoring and enhance the ability of NIRS-derived data to contribute to diagnosis in other clinical applications. 


\section{A- Asymptomatic}

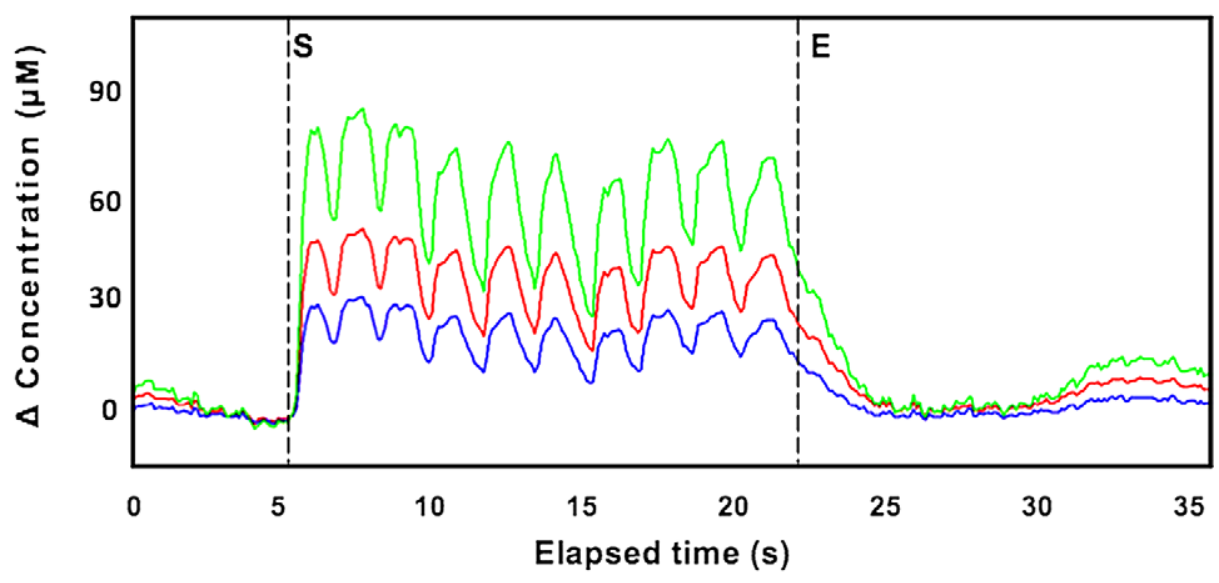

\section{B- Symptomatic}

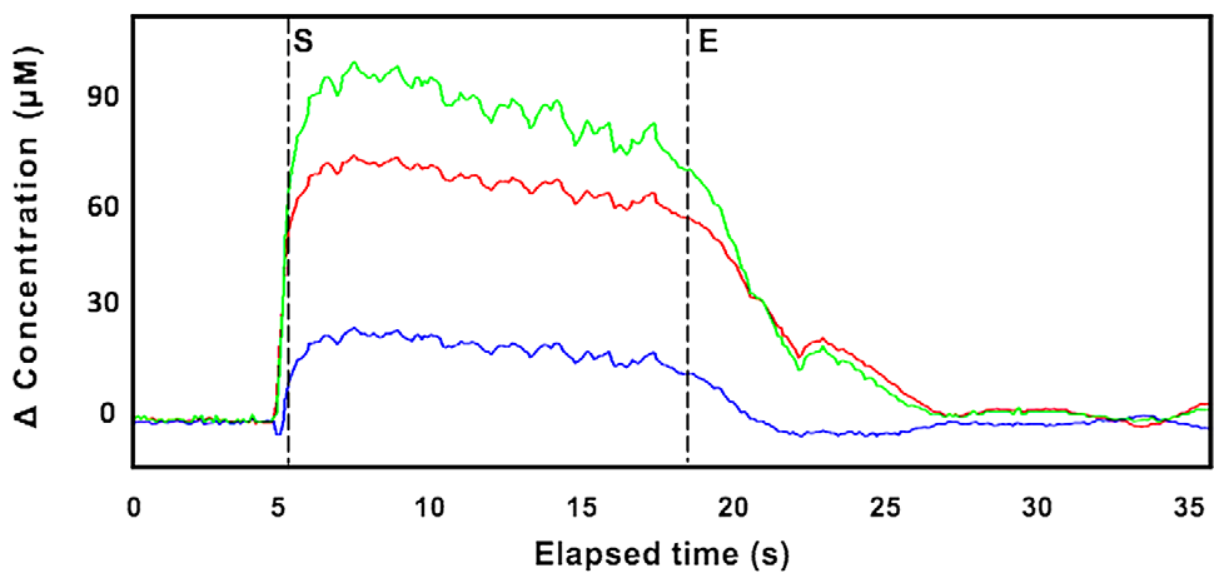

Total $\mathrm{Hb}$

Deoxygenated $\mathrm{Hb}$

Oxygenated $\mathrm{Hb}$

Fig. 2. Examples of characteristic graphs of change in $\mathrm{O}_{2} \mathrm{Hb}, \mathrm{HHb}$ and $\mathrm{tHb}$ during 10 repeated maximal pelvic floor contractions followed by relaxation in subjects with asymptomatic and symptomatic pelvic floor muscle dysfunction; both smoothed with 5 point averaging. $S$ indicates start of the contraction/relaxation sequence and $\mathrm{E}$ the end. Differences are seen in the magnitude of chromophore change and the slope of recovery of $\mathrm{O}_{2} \mathrm{Hb}$ (middle trace) on relaxation of the tenth contraction. There is also the impression of a negative trend in the concentration of both chromophores and $\mathrm{tHb}$ in symptomatic subjects. (Colors are visible in the online version of the article; http://dx.doi.org/10.3233/SPE-2010-0486.)

The superiority of CART classification to LDA in voiding studies is most probably because data from the entire time course of voiding is used. This suggests that the relationship between NIRS-derived changes in chromophore concentration and urodynamic-derived pressure-flow variables is complex and non-linear, because CART is a methodology able to classify populations divided by more complex bor- 
ders $[4,8,20]$. In addition, if the relationship were indeed simple or linear, generation of a nomogram using $[\Delta \mathrm{HHb}],\left[\Delta \mathrm{O}_{2} \mathrm{Hb}\right]$ should be possible that was equivalent to the Abrams-Griffiths nomogram, where the borders between regions would be straight lines. In this situation LDA would also give good results for these NIRS parameters at $Q_{\max }$, because this is the point used successfully for classification via the Abrams-Griffiths nomogram. However, as our data indicate this is not the case, which again implies a complex relationship between NIRS and urodynamic variables. This interpretation is also supported by the lack of benefit when latencies, slopes and standard deviations were used with both algorithms; the lack of good results that followed preprocessing of the data with both principal and independent component analysis; and $P_{\text {det }}$ being clearly superior for classification to $Q$.

While $P_{\operatorname{det}}$ is clearly the most influential urodynamic variable, clinical diagnosis of BOO based on this parameter is recognized to require an invasive procedure with associated risks and limited patient compliance [22]; hence the attraction of a non-invasive optical methodology. Urodynamic measurement also cannot measure the changes in oxygenation and hemodynamics that NIRS provides. Such changes are recognized to correlate with certain pathologies [15,23,32], hence NIRS could potentially offer a non-invasive diagnostic methodology for other causes of voiding dysfunction.

However, for NIRS monitoring to have any place in diagnostic evaluation reproducible classification methodology with appropriate accuracy is essential, as lack of reproducibility and specificity of NIRS data have compromised other potential clinical applications [9,23,26,35]. Importantly, CART analysis $[4,8,20]$ has met this criterion in its initial application to NIRS data, enabling patients to be classified for the presence or absence of bladder outlet obstruction [31], hence the logic and potential for investigators to explore using this method of classification in other NIRS monitoring applications.

We recognize limitations in this report; CART analysis was done retrospectively, not as the defined method of data analysis in the original prospective study; to date the algorithm developed has only been tested against a subset of the original data, not against new data; and the one urologic pathology classified using this methodology has been reported. However, CART has been applied to analyze various other data sets post hoc $[12,19,20]$; algorithm testing against a data subset alone is a valid option $[4,8,20]$ (although a new data set provides additional precision as the decision tree is pruned more effectively); we provide detail essential for CART analysis not able to be included in the original report [31] only using published data as explanatory examples.

If validated and refined by subsequent use, the application of CART analysis to NIRS monitoring we describe would represent an important clinical advance, as the algorithm using $[\Delta \mathrm{HHb}]$ and $\left[\Delta \mathrm{O}_{2} \mathrm{Hb}\right]$ offers a robust method for classifying subjects using non-invasive data alone. In addition, the differences evident in the pilot data included suggest other NIRS monitoring applications in children and women that appear suited to CART classification. However, the success of future studies exploring the ability of CART to discriminate between other pathologies will depend on the ability of researchers using NIRS to find the features in their data that allow classification with minimal error. We believe it is probable that CART was effective in the clinical context we investigated, because inclusion of NIRS data from the entire voiding time course allowed detection of statistical tendencies given by hidden features we are not yet able to identify. This situation may well apply to other NIRS data.

A fundamental goal for investigators using NIRS in a variety of applications has been to find a methodology that can reproducibly distinguish between patients with and without pathology. Consequently we have comprehensively described the principles of CART analysis and the process for diagnostic algorithm development, so that others using NIRS monitoring can consider applying this methodology. 


\section{References}

[1] Y.N. Bhambhani, Muscle oxygenation trends during dynamic exercise measured by near infrared spectroscopy, Can. J. Appl. Physiol. 29 (2004), 504-523.

[2] R. Boushel, R. Langberg, J. Olesen, J. Gonzales-Alonzo, J. Bulow and M. Kjaer, Monitoring tissue oxygen availability with near infrared spectroscopy (NIRS) in health and disease, Scand. J. Med. Sci. Sports 11 (2001), 213-222.

[3] R. Boushel and C.A. Piantadosi, Near-infrared spectroscopy for monitoring muscle oxygenation, Acta Physiol. Scand. 168 (2000), 615-622.

[4] L. Breiman, Classification and Regression Trees, Wadsworth International Group, Belmont, CA, USA, 1984, p. 358.

[5] P. Comon, Independent component analysis: a new concept?, Signal Processing 36(3) (1994), 287-314.

[6] R.O. Duda, P.E. Hart and D.G. Stork, Pattern Classification, Wiley, New York, 2001, p. 654.

[7] M. Ferrari, L. Mottola and V. Quaresima, Principles, techniques, and limitations of near infrared spectroscopy, Can. J. Appl. Physiol. 29(4) (2004), 463-487.

[8] A. Fielding, Cluster and Classification Techniques for the Biosciences, Cambridge Univ. Press, Cambridge, 2007, pp. 4068.

[9] G. Greisen, Is near-infrared spectroscopy living up to its promises?, Semin. Fetal. Neonatal. Med. 11(6) (2006), 498-502.

[10] T. Hald, Pathophysiology of the Urinary Bladder in Obstruction and Ageing, Health Publications, London, 1988, pp. 131138.

[11] T. Hamaoka, K.K. McCully, V. Quaresima, K. Yamamoto and B. Chance, Near-infrared spectroscopy/imaging for monitoring muscle oxygenation and oxidative metabolism in healthy and diseased humans, J. Biomed. Opt. 12(6) (2007), 062105 .

[12] K.R. Hess, M.C. Abruzzese, R. Lenzi, M.N. Raber and J.L. Abruzzese, Classification and regression tree analysis of 1000 consecutive patients with unknown primary carcinoma, Clin. Cancer Res. 5 (1999), 3403-3410.

[13] A. Hyvärinen and E. Oja, Independent component analysis: algorithms and application, Neural Netw. 13(4,5) $(2000)$, 411-430.

[14] C.S. Lim and P. Abrams, The Abrams-Griffiths nomogram, World J. Urol. 13 (1995), 34.

[15] A.J. Macnab, Biomedical applications of near-infrared spectroscopy, in: Biological and Biomedical Spectroscopy, Advances in Biomedical Spectroscopy, Vol. 2, A. Barth and P. Haris, eds, IOS Press, Amsterdam, 2009, pp. 355-402.

[16] A.J. Macnab, B. Shadgan, L. Stothers and K. Afshar, Ambulant monitoring of bladder oxygenation and hemodynamics using wireless near-infrared spectroscopy, J. Can. Urol. Assoc. (2011), in press.

[17] A.J. Macnab and L. Stothers, Development of a near-infrared spectroscopy instrument for applications in urology, Can. J. Urol. 15(5) (2008), 4233-4240.

[18] A.J. Macnab and L. Stothers, Near infrared spectroscopy: validation of bladder-outlet obstruction assessment using noninvasive parameters, Can. J. Urol. 15(5) (2008), 4241-4248.

[19] J. Mair, J. Smidt, P. Lechleitner, F. Dienstl and B. Puschendorf, A decision tree for the early diagnosis of acute myocardial infarction in non-traumatic chest pain patients at hospital admission, Chest 108 (1995), 1502-1509.

[20] R.J. Marshall, The use of classification and regression trees in clinical epidemiology, J. Clin. Epidemiol. 54 (2001), 603.

[21] S.P. Miller, J. Weiss, A. Barnwell, D.M. Ferriero, B. Latal-Hajnal, A. Ferrer-Rogers, N. Newton, J.C. Partridge, D.V. Glidden, D.B. Vigneron and A.J. Barkovich, Seizure-associated brain injury in term newborns with perinatal asphyxia, Neurology 58(4) (2002), 542-548.

[22] V.W. Nitti, Pressure flow urodynamic studies: the gold standard for diagnosing bladder outlet obstruction, Rev. Urol. 7(Suppl. 6) (2005), S14.

[23] J. Pannek, Editorial comment on: Classification of male lower urinary tract symptoms using mathematical modeling and a regression tree algorithm of noninvasive near-infrared spectroscopy parameters, Eur. Urol. 57(2) (2010), 332-333.

[24] C. Payer, B. Urlesberger, M. Pauger and W. Muller, Apnea associated with hypoxia in preterm infants: impact on cerebral blood volume, Brain Dev. 25(1) (2003), 25-31.

[25] P. Rolfe, In vivo near-infrared spectroscopy, Annu. Rev. Biomed. Eng. 2 (2000), 715-754.

[26] H. Sato, M. Kiguchi, A. Maki, Y. Fuchino, A. Obata, T. Yoro and H. Koizumi, Within-subject reproducibility of nearinfrared spectroscopy signals in sensorimotor activation after 6 months, J. Biomed. Opt. 11 (2006), 014021.

[27] B. Shadgan, K. Afshar, L. Stothers and A.J. Macnab, Near-infrared spectroscopy of the bladder: a new technique for studying lower urinary tract function in health and disease, in: Photonic Therapeutics and Diagnostics, N. Kollias et al., eds, Proc. SPIE, Vol. 7548, 2010, DOI: 10.1117/12.841066.

[28] B. Shadgan, W.D. Reid, R. Gharakhanlou, L. Stothers and A.J. Macnab, Wireless near-infrared spectroscopy of skeletal muscle oxygenation and hemodynamics during exercise and ischemia, Spectroscopy 23(5) (2009), 233-241.

[29] B. Shadgan, L. Stothers and A.J. Macnab, A transvaginal probe for near-infrared spectroscopic monitoring of the bladder detrusor muscle and urethral sphincter, Spectroscopy 22(6) (2008), 429-436.

[30] M.G. Sowa, L. Leonardi, J.R. Payette, K.M. Cross, M. Gomez anf J.S. Fish, Classification of burn injuries using nearinfrared spectroscopy, J. Biomed. Opt. 11 (2006), 054002. 
[31] L. Stothers, R. Guevara and A.J. Macnab, Classification of male urinary tract symptoms using mathematical modeling and a regression tree algorithm of non invasive near infrared spectroscopy parameters, Eur. Urol. 57(2) (2010), 327-333.

[32] L. Stothers, B. Shadgan and A.J. Macnab, Urologic applications of infrared spectroscopy (NIRS), Can. J. Urol. 15(6) (2008), 4399-4409.

[33] M.C. van der Sluijs, N.M.J. Colier, R.J.F. Houston and B. Oesberg, A new and highly sensitive continuous wave nearinfrared spectrophotometer with multiple detectors, Proc. SPIE 3194 (1997), 63-72.

[34] M. Wolf, M. Ferrari and V. Quaresima, Progress of near-infrared spectroscopy and topography for brain and muscle clinical applications, J. Biomed. Opt. 12(6) (2007), 062104.

[35] A.J. Wolfberg and A.J. du Plessis, Near-infrared spectroscopy in the fetus and neonate, Clin. Perinatol. 33 (2006), $707-$ 728. 


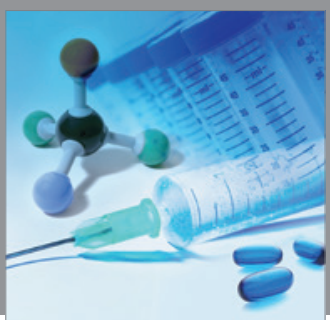

International Journal of

Medicinal Chemistry

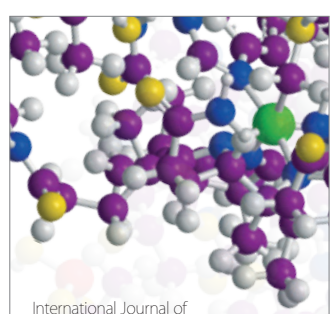

Carbohydrate Chemistry

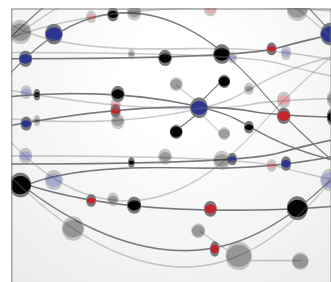

The Scientific World Journal
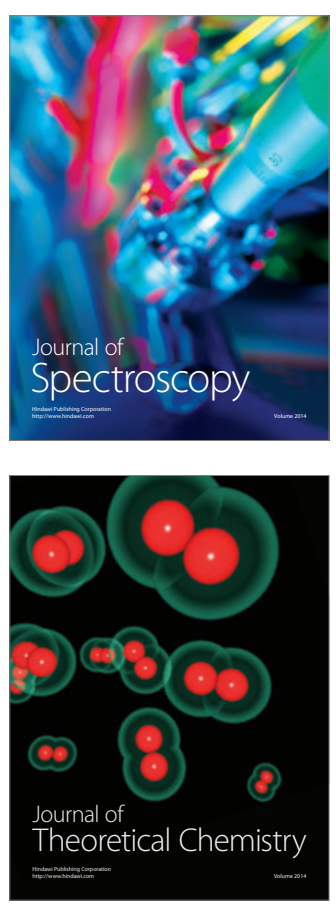
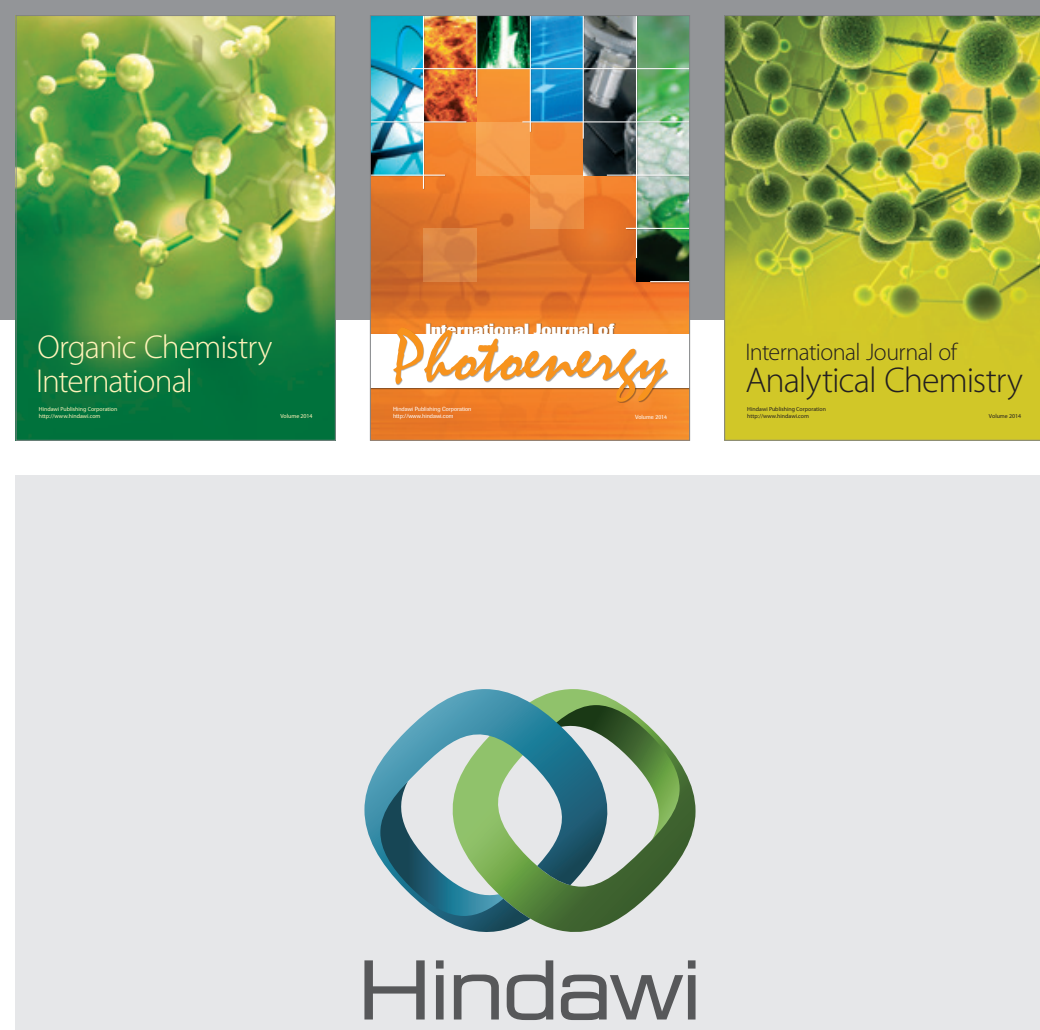

Submit your manuscripts at

http://www.hindawi.com
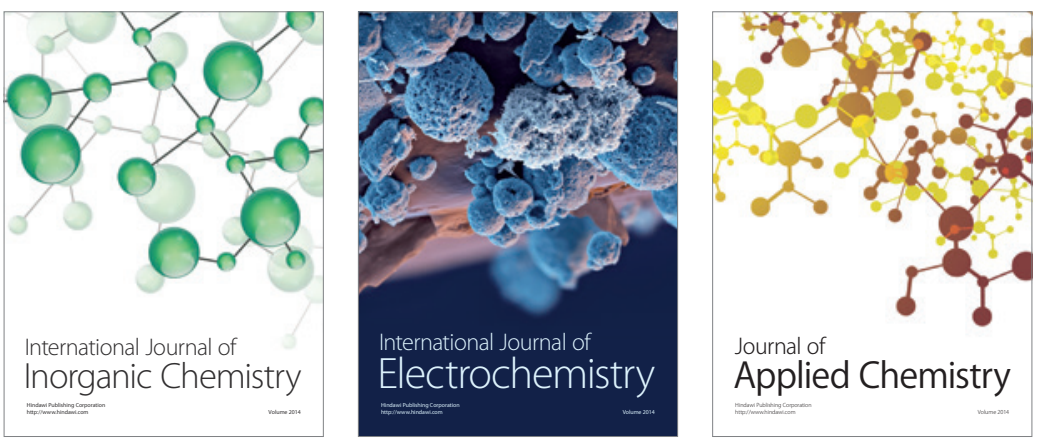

Journal of

Applied Chemistry
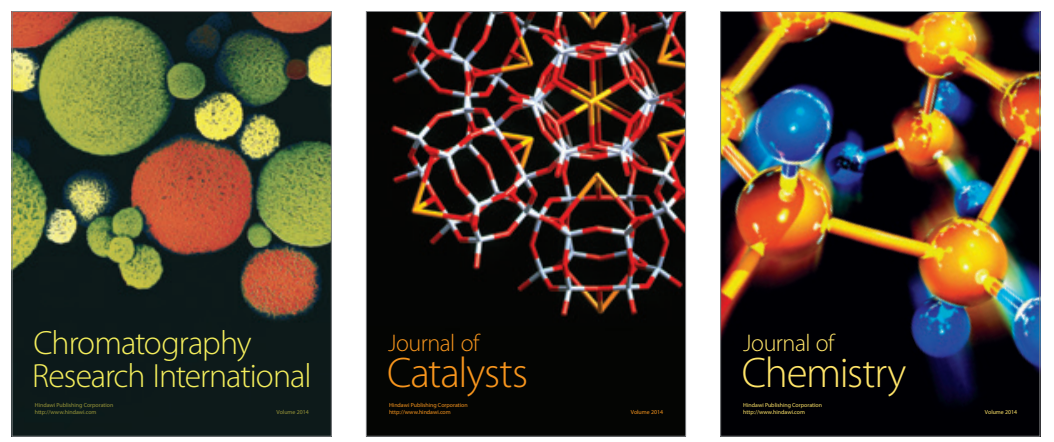
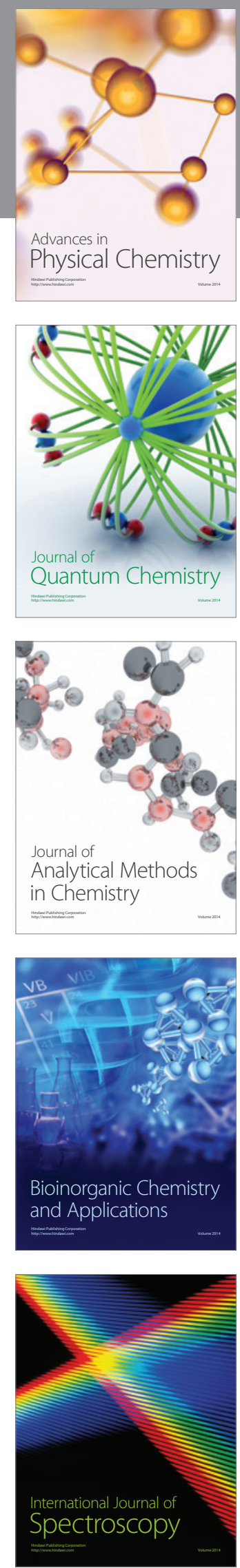